omitted. This Correspondence is signed in an individual capacity. The views and opinions expressed do not necessarily reflect that of any organization we (the authors) are associated with or employed by. This Correspondence does not represent the official view of the National Institute of Neurological Disorders and Stroke, the US National Institutes of Health, or any part of the US federal government. No official support or endorsement of this article by the National Institute of Neurological Disorders and Stroke or the US National Institutes of Health is intended or should be inferred. All authors have read the Nature Research Authorship Policy and confirm that this Correspondence complies.
Author contributions

E.L.L., E.T.I. and A.I.A conceived of and drafted the manuscript; and all authors edited and revised the manuscript and approved the final version of the manuscript.

\title{
Accelerating the translational medicine cycle: the Academia Europaea pilot
}

To the Editor-Translational Medicine (TM) is among the main challenges of the 21 st century; its development is essential for the application of scientific results for community benefit.

Although several developments have been achieved in the field, there is still room for improvement in implementing scientific results in healthcare. More than 1.4 million articles are published each year, as reported on PubMed; however, much of this knowledge is not applied in everyday practice. Based on a report from the European Commission's European Statistical Office, around 1.7 million people under 75 years of age die in Europe every year, but 1.2 million of these deaths could have been avoided through effective primary prevention and public-health intervention ${ }^{1}$.

The Academia Europaea, one of the leading advisory bodies of the European Commission, thus initiated the development of a new TM model that facilitates and accelerates the application of scientific knowledge for community benefit ${ }^{2}$. The new cycle model equally focuses on healthcare, the acquisition of new scientific findings, the digestible summation of results, and the communication of scientific knowledge to all stakeholders, including patients, healthcare professionals, pharmaceutical companies and policymakers. The model has been piloted at the Centre for Translational Medicine at the University of Pécs (UP-CTM) in Hungary, with great success, which made UP-CTM one of the most efficient and visible centers for TM in Eastern and Central Europe within five years.

The TM cycle model in healthcare was trialed in an eight-bed UP-CTM patient care unit that focused on managing acute pancreatitis. Patient coordinators, clinical research administrators, $\mathrm{PhD}$ students, nurses, and junior and senior doctors joined forces in the day-to-day work. This team ensured the application of available evidence-based guidelines and, notably, built up activities for healthcare delivery science-for example, the team enrolled patients in clinical trials and patient registries, and organized and analyzed the diagnostic and treatment pathways. In a year, we achieved a two-day reduction in the length of hospitalization per patient, a marked decrease in antibiotics administration, and a two-thirds drop in mortality. Due to the healthcare-delivery-science activity, patient-care costs also fell by $25 \%$ (ref. ${ }^{3}$ ) (Fig. 1a).

The model was subsequently tested in two other hospitals. At the Szent György University Teaching Hospital of Fejér County (Székesfehérvár, Hungary), a similar healthcare delivery science-based patient care unit for acute pancreatitis was launched. As a result, the use of antibiotics substantially decreased. The duration of hospitalization dropped by an average of one day per patient (Fig. 1b). At the Heim Pál National Pediatric Institute (Budapest, Hungary), a scientifically constructed cystic-fibrosis registry, which also examined glucose metabolism in detail, facilitated the early diagnosis of diabetes mellitus in $14 \%$ of the patients; these would have been missed by the previous system. In addition, nine children were identified as having impaired glucose tolerance; this may allow earlier diagnosis and treatment of established cystic fibrosis-related diabetes.

After the projects involving acute pancreatitis, we broadened our patient registries and clinical trials to a wide range of medical disciplines (Fig. 1c,d). Notably, these clinical databases and biobank samples substantially added to the 300 publications by UP-CTM over the five years, which may substantially contribute to the implementation of scientific results for community benefit. The outstanding networking is well illustrated through UP-CTM's joint publications involving more than a thousand researchers in the five years (Fig. 1e).

To support patient care, scientific activity and communication, we set up an interdisciplinary unit at the outset with several different disciplines, including patient coordination, biostatistics, informatics, data management, artificial intelligence, legal support and communication. Notably, the unit accelerated scientific activity, supported patient care and communication, and created 51 jobs with new professional content by the end of the five years, which is extremely important in a century during which the demand for professionals is decreasing in numerous fields (Fig. 1f).

A shift was necessary for the academic field and communication as well. Medical professionals must understand scientific results to deliver high-quality patient care; therefore, our center developed a new 'learning by doing' education module in which 45 young physicians learned the scientific methodology. The program's strength is emphasized by the fact that doctors from ten different cities in four countries participated in the training program. At the same time, we developed a complex Clinical Research Administrator training program accredited and tested in the fifth year. During this period, printed educational materials and online educational videos were made available for healthcare professionals; these have been particularly useful during the COVID-19 pandemic. Grant committees also recognized the potential of the TM cycle approach (Fig. 1g). 

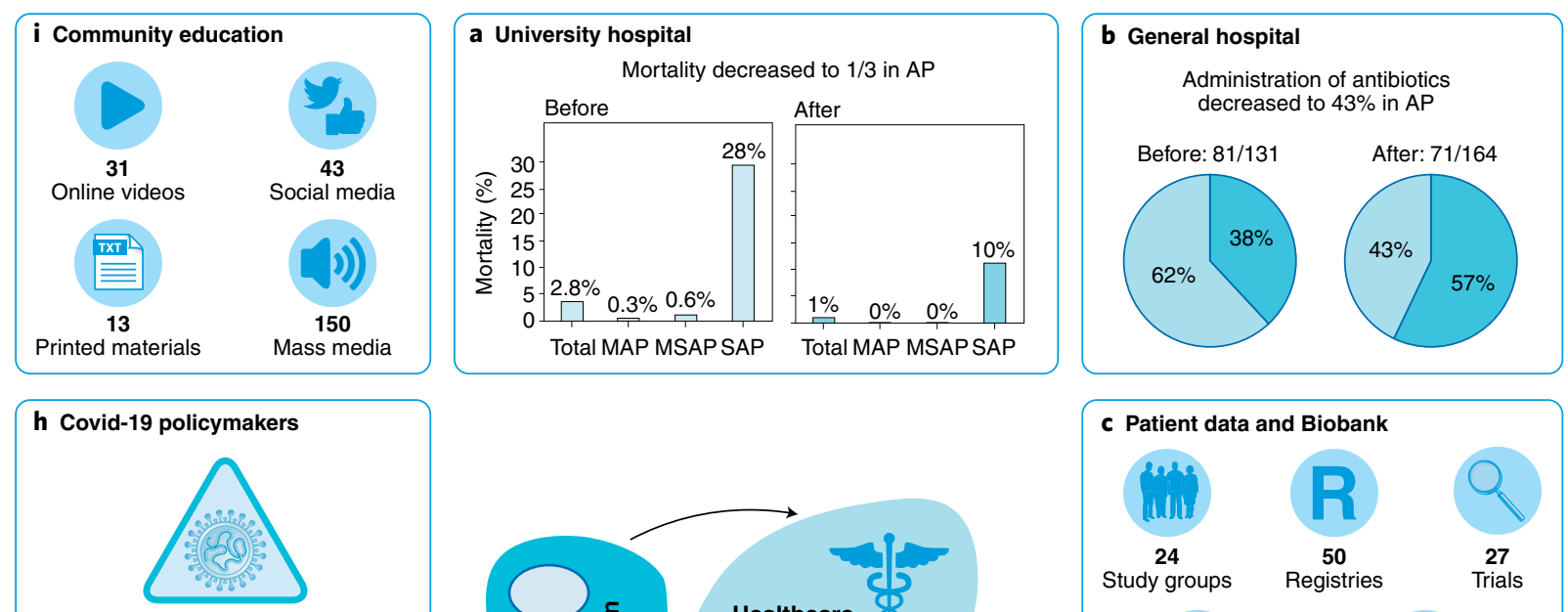

2

Extensive reports were presented directly to the national epidemiologica policymaking body during the first wave
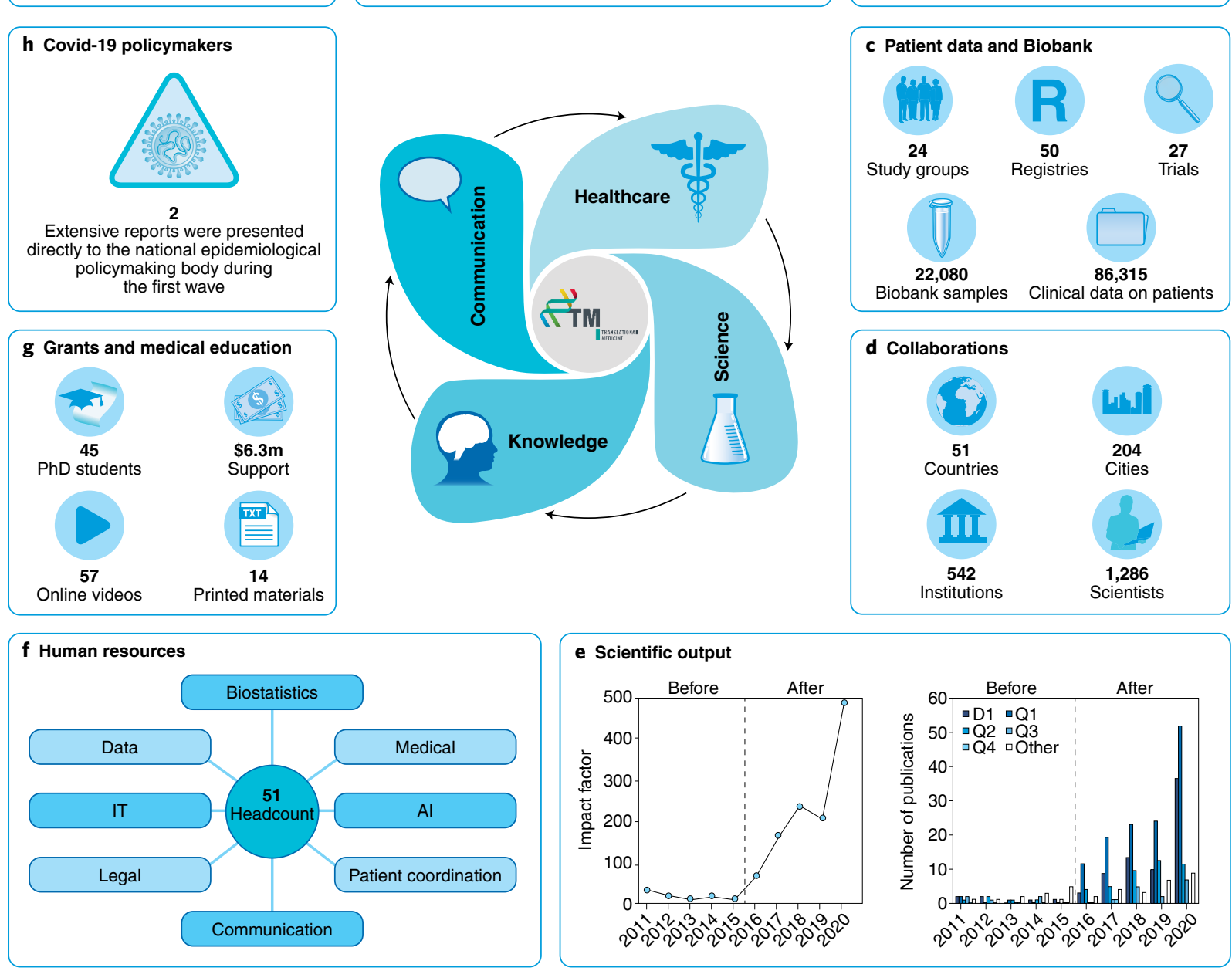

Fig. 1 | Five-year results of implementation of the Academia Europaea TM cycle model. a, Total mortality and mortality from moderate acute pancreatitis (MAP), moderately severe acute pancreatitis (MSAP) and severe acute pancreatitis (SAP) before (left) and after (right) implementation of the UP-CTM patient care unit. $\mathbf{b}$. Antibiotics use for acute pancreatitis before and after implementation of the UP-CTM patient care unit; pie charts indicate the proportion of patients who received antibiotics (light blue) or not (teal). c,d, Clinical databases and biobank samples (c) and collaborations (d) included in the UP-CTM after the initial five-year period. e, Yearly scientific output (horizontal axes) before and after implementation of the UP-CTM. D1, publications in the top 10\% of the journals; Q1-Q4, publications in the first quartile (top 25\%) and second, third and fourth quartiles of the journals, respectively. f, Components of the interdisciplinary unit of the UP-CTM. IT, information technology; Al, artificial intelligence. $\mathbf{g}$, Educational efforts and support of the UP-CTM. h,i, Outreach of the UP-CTM to policymakers (h) and the community (i) during the COVID-19 pandemic.

For the best implementation of scientific results in practice, information must also be disseminated to non-medical people. With the onset of the COVID-19 pandemic, we immediately established the Translational Action and Research Group against Coronavirus (KETLAK) to reduce the coronavirus-induced health damage and deaths. Two extensive reports were presented directly to Hungary's national epidemiological policymaking body by our KETLAK group in April 2020, which facilitated the country's management of the first wave of the COVID-19 pandemic with excellent results 4 . We launched the PROACTIVE-19 study, providing health education to the community from the outbreak of this pandemic ${ }^{5}$. We had approximately 150 media appearances, which also played an essential role in communication to the community (Fig. 1h). 
Of course, we concentrated not only on COVID-19 but also on other diseases. In total, we helped the population with 31 patient-education videos, 43 social-media appearances and 13 printed patient-information leaflets (Fig. 1i).

What does the future hold? It is hard to predict, but it is certainly up to us. We strongly believe that disseminating the model and highlighting the successes it achieves can help. It is an excellent sign that Semmelweis University has already invited us to build the TM cycle in Budapest; moreover, several European and American universities have already expressed interest in introducing the TM cycle model or further developing their existing models. Is this model perfect? Certainly not, but it represents a considerable advance.

\footnotetext{
Péter Hegyi ${ }^{1,2,3}$, Bálint Erőss ${ }^{1,2}$,

Ferenc Izbéki ${ }^{4 凶}$, Andrea Párniczky ${ }^{1,2,5}$ and Andrea Szentesi $\mathrm{i}^{1,2,3 凶}$

${ }^{1}$ Institute for Translational Medicine, Szentágothai Research Centre, Medical School, University of Pécs, Pécs, Hungary. ${ }^{2}$ Centre for Translational Medicine, Semmelweis University, Budapest, Hungary. ${ }^{3}$ Centre for Translational Medicine, First Department of Medicine, University of Szeged, Szeged, Hungary. ${ }^{4}$ Szent György University Teaching Hospital of Fejér County, Székesfehérvár, Hungary. ${ }^{5}$ Heim Pál National Pediatric Institute, Budapest, Hungary.
}

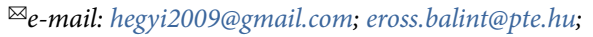
fizbeki@gmail.com; andrea.parniczky@gmail.com;

$\sqsupset$ szentesiai@gmail.com

Published online: 26 July 2021

https://doi.org/10.1038/s41591-021-01458-8

References

1. OECD \& European Observatory on Health Systems and Policies. OECDiLibrary https://www.oecd-ilibrary.org/ social-issues-migration-health/hungary-country-health-profile2019_4b7ba48c-en (28 November 2019).

2. Hegyi, P. et al. J. Clin. Med. 19, 1532 (2020).

3. Godi, S. et al. J. Gastrointestin. Liver Dis. 27, 151-157 (2018).

4. Gombos, K. et al. Popul. Health Manag. 24, 35-45 (2021).

5. Eross, B. et al. Trials 21, 809 (2020).

The authors declare no competing interests.

\section{A French cohort for assessing COVID-19 vaccine responses in specific populations}

To the Editor-The COVID-19 vaccination campaign started in France on 27 December 2020. It has been rolled out in different priority phases according to the risk of developing a severe form of COVID19 and the risk of being exposed to the causative coronavirus SARS-CoV-2. By 5 May 2021, four vaccines against COVID19 were approved by the European Medicines Agency and were available in France: COMIRNATY (the COVID-19 mRNA vaccine BNT162b2; BioNTechPfizer); COVID-19 Vaccine Moderna (mRNA-1273; Moderna); VAXZEVRIA (ChAdOx1-nCoV19; AstraZeneca-Oxford University); and COVID-19 Vaccine Janssen (Ad26.COV2.S; Janssen). Specific populations are defined as people at risk of developing severe forms of the disease and in whom the immunogenicity and efficacy of vaccines against that disease may differ from that of the general population (e.g., recipients of solid-organ transplants or patients undergoing hemodialysis). The safety, immunogenicity and efficacy of vaccines in specific populations, which are heterogeneous groups of patients, are affected by the nature and intensity of the underlying disease(s), the age of the patient and any other treatments the patient is taking, and are possibly affected by the vaccine platform used. So far, no or only limited data on specific populations are available from published results of phase 3 trials of authorized vaccines against
COVID-19. Initial immunogenicity data available for some of these specific populations showed low antibody responses to the SARS-CoV-2 spike protein in patients who received solid-organ transplantation ${ }^{1-4}$, patients undergoing hemodialysis ${ }^{5,6}$, patients receiving chemotherapy or immunotherapy for solid cancer or hematologic malignancies ${ }^{7,8}$, and patients receiving infliximab for inflammatory bowel disease ${ }^{9}$. Most of these studies reported small sample sizes.

To assess the immune response of COVID-19 vaccines in different specific populations, INSERM (Institut National de la Santé et de la Recherche Médicale) and ANRS-MIE (Agence Nationale de Recherche sur le Sida-Maladies Infectieuses Emergentes), in collaboration with the COVIREIVAC network, ten national disease-specific societies and seven patients' associations (France Rein, Transhépate, ARSEP Foundation, CNAO, FFD, EGMOS and TRT5 CHV), launched, on 25 March 2021, the ANRS0001S COV-POPART study (ClinicalTrials. gov NCT04824651). COV-POPART is a national multi-center prospective multi-cohort study of specific populations vaccinated against COVID-19 that aims to include 10,700 patients.

Patients with solid cancer $(n=800)$, solid-organ transplantation $(n=700)$, hematopoietic stem cell transplantation $(n=350)$, chronic renal failure with or without dialysis $(n=350)$, multiple sclerosis or neuromyelitis optica spectrum disorders $(n=600)$, autoimmune inflammatory rheumatic diseases $(n=600)$, systemic autoimmune diseases $(n=600)$, hypogammaglobulinemia $(n=300)$, obesity $(1,400)$, diabetes mellitus $(n=1,400)$ and/ or infection with human immunodeficiency virus $(n=1,400)$ will be included. All adults affected by at least one of these chronic conditions, without a history of COVID-19 and not yet vaccinated, will be included in one of the 35 participating centers, which represent more than 250 clinical sites. A control group without any of the above-mentioned underlying conditions will also be included $(n=1,850 ; 18-74$ years of age $(n=1,400)$ and $\geq 75$ years of age $(n=450))$.

The main objective will be to analyze the humoral immune response by assessing IgG antibody to the SARS-CoV-2 spike protein (by ELISA), IgG antibody to the SARS-CoV-2 receptor-binding domain (by ELISA) and specific neutralizing antibody to SARS-CoV-2 (by classical in vitro neutralization assay) at 1 month, 6 months, 12 months and 24 months after the first dose (Janssen vaccine) or second dose (all other vaccines) of the vaccine regimen (Fig. 1). Secondary objectives will be to compare the kinetics and strength of the immune responses of each subpopulation (e.g., recipients of solid-organ transplantation) with those of the control group and to 